\title{
Mechanism of Dihydromyricetin on Inflammatory Diseases
}

\author{
Yang Sun ${ }^{1}$, Shasha Liu ${ }^{2}$, Songwei Yang ${ }^{1}$, Chen Chen $^{3}$, Yantao Yang ${ }^{1}$, Meiyu Lin ${ }^{1}$, Chao Liu ${ }^{4}$, \\ Wenmao Wang ${ }^{4}$, Xudong Zhou ${ }^{5}$, Qidi Ai ${ }^{1 *}$, Wei Wang ${ }^{5 *}$ and Naihong Chen ${ }^{1,6 *}$

\begin{abstract}
${ }^{1}$ Hunan Engineering Technology Center of Standardization and Function of Chinese Herbal Decoction Pieces and College of Pharmacy, Hunan University of Chinese Medicine, Changsha, China, ${ }^{2}$ Pharmacy Department, Xiangtan Central Hospital, Xiangtan, China, ${ }^{3}$ Department of Pharmacy, The First Hospital of Lanzhou University, Lanzhou, China, ${ }^{4}$ Zhangjiajie Meicha Technology Research Center, Hunan Qiankun Biotechnology Co., Ltd, Zhangjiajie, China, ${ }^{5}$ TCM and Ethnomedicine Innovation and Development International Laboratory, Innovative Materia Medica Research Institute, School of Pharmacy, Hunan University of Chinese Medicine, Changsha, China, 'SState Key Laboratory of Bioactive Substances and Functions of Natural Medicines, Institute of Materia Medica and Neuroscience Center, Chinese Academy of Medical Sciences and Peking Union Medical College,
\end{abstract} \\ Beijing, China
}

Inflammation plays a crucial role in a variety of diseases, including diabetes, arthritis, asthma, Alzheimer's disease (AD), acute cerebral stroke, cancer, hypertension, and myocardial ischemia. Therefore, we need to solve the problem urgently for the study of inflammation-related diseases. Dihydromyricetin (DHM) is a flavonoid mainly derived from Nekemias grossedentata (Hand.-Mazz.) J.Wen and Z.L.Nie (N.grossedentata). DHM possesses many pharmacological effects, including anti-inflammatory (NLRP-3, NF- $\mathrm{BB}$, cytokines, and neuroinflammation), antioxidant, improving mitochondrial dysfunction, and regulating autophagy and so on. In this review, we consulted the studies in the recent 20 years and summarized the mechanism of DHM in inflammation-related diseases. In addition, we also introduced the source, chemical structure, chemical properties, and toxicity of DHM in this review. We aim to deepen our understanding of DHM on inflammation-related diseases, clarify the relevant molecular mechanisms, and find out the problems and solutions that need to be solved urgently. Providing new ideas for DHM drug research and development, as well as broaden the horizons of clinical treatment of inflammation-related diseases in this review. Moreover, the failure of clinical transformation of DHM poses a great challenge for DHM as an inflammation related disease.

Keywords: inflammation, neuroinflammation, DHM, ampelopsisgrossedentata, mechanism

\section{INTRODUCTION}

Dihydromyricetin (DHM) is a flavonoid mainly derived from Nekemias grossedentata (Hand.Mazz.) J.Wen and Z.L.Nie (N.grossedentata) (Liu et al., 2019a). Flavonoids have multifarious pharmacological effects, such as antioxidant, anti-inflammatory response, anti-cancer, and antiviral as well as neuroprotective effects (Wang et al., 2016a). DHM exists not only in N. grossedentata, but also in plant food (Wu et al., 2015). There are many inflammatory diseases that affect peoples' physical and mental health as well as the quality of life. For instance, atherosclerosis (Geovanini and Libby, 2018), diabetic cardiomyopathy (Li et al., 2019), endothelial dysfunction (Bai et al., 2020), neurodegenerative diseases (Stephenson et al., 2018), and cancer (Diakos et al., 2014) as well as liver disease (Yang et al., 2019), and so forth. In addition, DHM has numerous biological effects, including anti-oxidation, improving mitochondrial dysfunction, and regulating autophagy (Liu et al., 2020), 


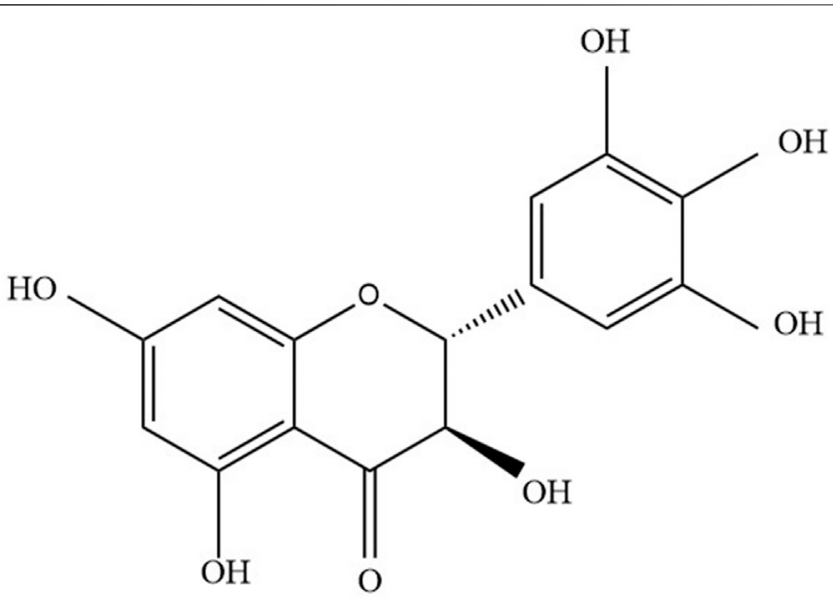

FIGURE 1 | The chemical structure of DHM.

especially anti-inflammatory effects (Zhang et al., 2018). This suggests that DHM exerts its pharmacological effects through the corresponding molecular mechanisms.

\section{N. GROSSEDENTATA}

$N$. grossedentata has been used as an herbal medicine belonging to the Parthenocissus inserta $f$. dubia (Rehder) Rehder J. Arnold Arbor (Ma et al., 2020) in China for thousands of years. "Compendium of Vatica rassak (Korth.) Blume Medica" believed that it had the effect of "regulating the middle and replenishing qi, promoting blood and promoting qi" (Hu et al., 2020), which was used as tea (Zhou et al., 2014). It is generally known as that Calamus rotang L. tea is used as a heat clearingherb in traditional Chinese medicine to promote diuresis and blood circulation (Huang et al., 2018). In addition, $N$. grossedentata is common in South China and can also be eaten (Jia et al., 2020). The leaves (Li et al., 2020) and stems (Gao et al., 2017) of the N. grossedentata are also known as vine tea. Its tender stems and leaves are widely used as Citrullus colocynthis (L.) Schrad. tea. It has been used for herbal tea and traditional Chinese medicine for hundreds of years. Modern pharmacological studies have shown that N. grossedentata has a variety of pharmacological effects, including antioxidative, antiinflammatory, and antiviral (Chen et al., 2016) as well as antithrombotic (Sun et al., 2020). Therefore, N. grossedentata treated many diseases clinically, such as diabetes (Chen et al., 2016), pharyngitis, sore throat, and fever associated with colds (Hou et al., 2015a).

\section{DIHYDROMYRICETIN}

DHM (3', 4', 5, 5', 7-Hexahydroxy-2, 3-dihydroflavanonol) was first discovered from Nekemias meliaefolia in 1940 (Zhang et al., 2001) (Figure 1). Therefore, DHM is also known as Ampelopsis japonica (Thunb) Makino (AMP) (Liu et al., 2020). The content of DHM in N. grossedentata was as high as 30-40\% (Liu et al., 2019a). DHM was also found in Vitis vinifera L., Myrica cerifera L., Prunus amygdalus Batsch, Ginkgo biloba L., and other plants (Liu et al., 2020). In addition, studies have confirmed the existence of DHM in Hovenia dulcis Thunb and Cedrus deodara (Roxb. ex D.Don) G.Don (Liang et al., 2014a; Liang et al., 2014b). DHM will degrade in a weak alkaline environment, especially under the condition of $\mathrm{Cu}^{2+}$ and $\mathrm{Fe}^{3+25}$. DHM was poorly soluble, only soluble in hot water and ethanol (Liu et al., 2019b). The efficacy of DHM will be affected by its bioavailability (Feng et al., 2018). The animal experimental data showed that the bioavailability of DHM in rats was $4.02 \%$ (Liu et al., 2017a). The main metabolic sites of DHM are in the liver and gastrointestinal tract, and some are absorbed by the blood. Following being absorbed by the blood, DHM can be distributed throughout the body (Ding et al., 2021). DHM was characterized by low bioavailability and unstable chemical properties, which limited the pharmacology and clinical application of DHM (Tong et al., 2015). And for DHM, only a few kinds of studies can conclude that DHM is not toxic (Zhang et al., 2014).

\section{INFLAMMATION AND INFLAMMATORY DISEASES}

Following sterile tissue injury, the positive response of the host to pathogens is called "inflammation" (Alessandri et al., 2013). Inflammation is divided into acute inflammation and chronic inflammation (Arulselvan et al., 2016). When inflammation is triggered, it leads to the recruitment and activation of neutrophils, monocytes, macrophages, and other immune cells ( $\mathrm{He}$ et al., 2020). Macrophages are the first immune cells affected by the inflammatory response (Lopez-Castejon, 2020). In addition, the inflammatory process is regulated by cytokines. Cytokines are secreted by immune cells (Kirkpatrick and Miller, 2013). Inflammation is a multi-stage and complex process, involving a variety of cells as well as signal cascades (Cas et al., 2020). The types of inflammation include acute inflammation and chronic inflammation (Arulselvan et al., 2016). In recent years, inflammation has been a research hotspot. Studies have shown that there is a complex relationship between inflammation and inflammatory disease. For example, atherosclerosis, heart failure (Shirazi et al., 2017), rheumatoid arthritis (Dhingra and Chopra, 2020), neurodegenerative diseases (Baune, 2015), cancer (Sgambato and Cittadini, 2010), and cardiac arrhythmogenesis (Yalta and Yalta, 2018). In general, many diseases are associated with inflammation, so we must pay attention to it.

\section{ANTI-INFLAMMATORY MECHANISM OF DHM}

\section{NLRP-3 and Pyroptosis}

Some studies have confirmed that DHM is closely related to palmitic acid (PA)-induced human umbilical vein endothelial cells (HUVECs). Hu et al. have clarified that DHM ameliorated pyroptosis by activating the Nrf2 (NF-E2-related factor 2) 
signaling pathway. DHM inhibited the activation of NLRP-3 by down-regulating mitochondrial reactive oxygen species (ROS) in PA-induced HUVECs (Hu et al., 2018). DHM treatment inhibited the activation of caspase- 1 and the expression of IL- $1 \beta$ in the brain of AD mice. This suggested that DHM inhibited the activation of the NLRP-3 signaling pathway to improve the inflammatory response in AD (Liu et al., 2019b). In addition, DHM improved pyroptosis caused by NLRP-3 in chronic liver injury mice (Cheng et al., 2020). DHM significantly inhibited cholesterol accumulation and foam cell formation, improved mitochondrial function, reduced oxidative stress as well as reduced the activation of NLRP3 in oxidized low-density lipoprotein (ox-LDL)stimulated macrophages in Sirtuin3 (SIRT3) ko mice (Sun et al., 2021). Studies have shown that NLRP-3 activation contributed to the development of cardiotoxicity. What is more, DHM improved myocardial injury by inhibiting the activation of NLRP-3 ${ }^{20}$.

\section{Nuclear Factor-кB (NF-кB)}

Research confirmed that DHM down-regulated the expression of the NF- $\kappa \mathrm{B}$ signal pathway by directly binding to I $\mathrm{B}$ kinase (IKK), thereby inhibiting IKK phosphorylation. To sum up, DHM exerted an antiarthritic effect in collagen-induced arthritis rats through down-regulation of NF- $\mathrm{KB}$ (Wu et al., 2019a). Tang et al. confirmed by Western blotting that DHM inhibited tumor necrosis factor- $\alpha$ (TNF- $\alpha$ )-induced phosphorylation of $\mathrm{IKK} \alpha / \beta$ dose-dependently. The results suggested that DHM significantly inhibited the expression of $\mathrm{NF}-\mathrm{kB}$, and then inhibited the inflammatory response (Tang et al., 2016). DHM inhibited the activation of macrophage by suppressing NF- $\kappa \mathrm{B}$ p65 phosphorylation, IKK $\beta$ activity, and IKK $\alpha / \beta$ phosphorylation in the NF- $\kappa B$ pathway (Wang et al., 2016b). Similarly, the research results of $\mathrm{Wu}$ et al. showed that DHM inhibited the activation of the NF- $\kappa B$ pathway by attenuating the phosphorylation of the NF$\kappa \mathrm{B}$ in rheumatoid arthritis (Wu et al., 2020).

\section{Cytokines}

Liu et al. have confirmed that DHM improved the inflammatory response of the liver and aorta by inhibiting the expression of TNF- $\alpha$ as well as Interleukin (IL)-6 in LDL receptor-deficient mice (Liu et al., 2017b). DHM reduced oxidative stress and downregulated the levels of TNF- $\alpha$, IL- 6, IL- $1 \beta$, and cyclooxygenase- 2 (COX-2) by activating Nrf2 in rheumatoid arthritis rat (Chu et al., 2018). Studies have confirmed that there is a close relationship between inflammation and the liver damage. DHM prevented TNF- $\alpha$ mediated liver toxicity by inhibiting the expression of TNF- $\alpha$ through the c-Jun N-terminal kinase (JNK) signaling pathway (Xie et al., 2015). Wu et al. have explained that the levels of IL- 6 and TNF- $\alpha$ in diabetic cardiomyopathy mice treated with DHM were significantly reduced (Wu et al., 2017). In addition, DHM inhibited the expression of inflammatory factors (IL-6 and TNF- $\alpha$ ) in rats with pulmonary hypertension (PH) (Li et al., 2017). DHM inhibited the expression of IL-4, IL-5, and IL-13 in alveolar lavage fluid in asthmatic mice (Xu et al., 2017).

\section{Neuroinflammation}

Neuroinflammation plays a significant role in many neurological diseases, such as depression (Troubat et al., 2021), AD (Calsolaro and Edison, 2016), stroke (Jayaraj et al., 2019), Parkinson's disease (PD) (Kustrimovic et al., 2019), amyotrophic lateral sclerosis (ALS) (Liu and Wang, 2017), and so on. DHM down-regulated the level of cytokines via NLRP-3 signaling pathway in AD mice (Liu et al., 2019a). Studies have confirmed that DHM effectively improved astrocyte and microglia mediated neuroinflammation (Wu et al., 2019b). DHM inhibited neuroinflammation in $\mathrm{AD}$ rats through adenosine $5^{\prime}$-monophosphate activated protein (AMPK)/signal transducer and activator of transcription 1 (SIRT1) pathway (Liu et al., 2020). DHM improved depressive symptoms by alleviating neuroinflammatory response (Ren et al., 2018). DHM inhibited the inflammatory response, inhibited the secretion of inducible nitric oxide synthase (iNOS) and COX-2, and attenuated the activation of NF- $\mathrm{BB}$ and TLR4 signals in lipopolysaccharide (LPS)-induced neuroinflammation (Jing and Li, 2019). DHM inhibited inflammatory responses via up-regulation of the AMPK/SIRT1 pathway in AD mice (Sun et al., 2019) (Figure 2).

\section{OTHER MECHANISMS OF DHM EXCEPT FOR ANTI-INFLAMMATORY}

Furthermore, DHM reduced inflammatory response via the JNK pathway in acute liver injury mice (Wang et al., 2018a). DHM reversed the metabolic syndrome by upregulating insulin receptor substrate-1 (IRS-1) (y612) tyrosine phosphorylation and improving insulin resistance in obese mice ( $\mathrm{He}$ et al., 2019). Of note, DHM inhibited the production of melanin by down-regulating the protein kinase $\mathrm{A}$ (PKA), protein kinase $\mathrm{C}$ (PKC), and mitogen-activated protein kinases (MAPK) signaling pathways in B16F10 cells (Huang et al., 2016). DHM inhibited the growth of Staphylococcus aureus by destroying the integrity of the cell wall and increasing the permeability of the cell membrane (Liang et al., 2020). Studies have shown that DHM promoted SIRT3 in chondrocytes via the AMPK-peroxisome proliferatoractivated receptor $\gamma$ coactiva-tor-1 (PGC-1 $\alpha$ )-SIRT3 signaling pathway in osteoarthritis rat (Wang et al., 2018b). DHM significantly reversed cisplatin-induced nephrotoxicity by reducing oxidative stress and inhibiting apoptosis (Wu et al., 2016). Studies have shown that DHM down-regulated microRNA-34a (miR-34a) in renal tubular epithelial cells by inhibiting the phosphorylation of p53 (tumor suppressor gene) induced by transforming growth factor $\beta 1$ (TGF- $\beta 1$ ) (Liu et al., 2019c). In addition, DHM ameliorated memory impairment caused by DHM and improves memory impairment caused by hypobaric hypoxia (Liu et al., 2016). DHM improved oxidative stress by inhibiting ROS production and increasing nitric oxide (NO) production in endothelial cells (Hou et al., 2015b). DHM reduced the production of inflammatory factors in mast cells by inhibiting signal transducer and activator of transcription 5 (STAT5) and the NF- $\mathrm{KB}$ signaling pathways. DHM improved mast cell proliferation by significantly attenuating IgE-induced ROS and inhibiting STAT5 phosphorylation in mast cells (Chang 


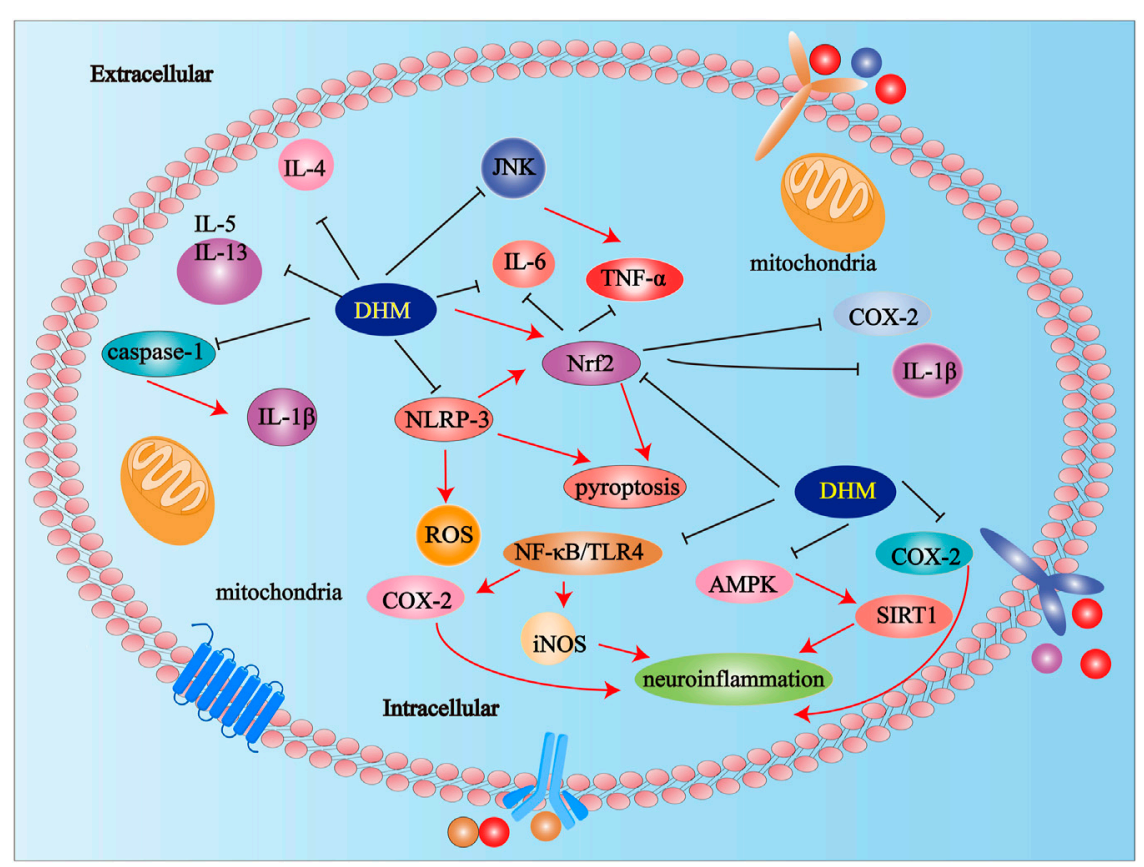

FIGURE 2 |Anti-inflammatory mechanisms of DHM. Abbreviations: IL-5, Interleukin-5; IL-13, Interleukin-13; IL-4, Interleukin-4; DHM, Dihydromyricetin; Interleukin$1 \beta$; Nrf2, NF-E2-related factor 2; TNF- $\alpha$, tumor necrosis factor; COX-2, cyclooxygenase-2; JNK, c-Jun N-terminal kinase; iNOS, inducible nitric oxide synthase; STAT,

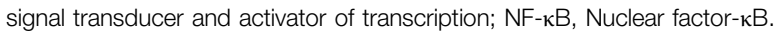

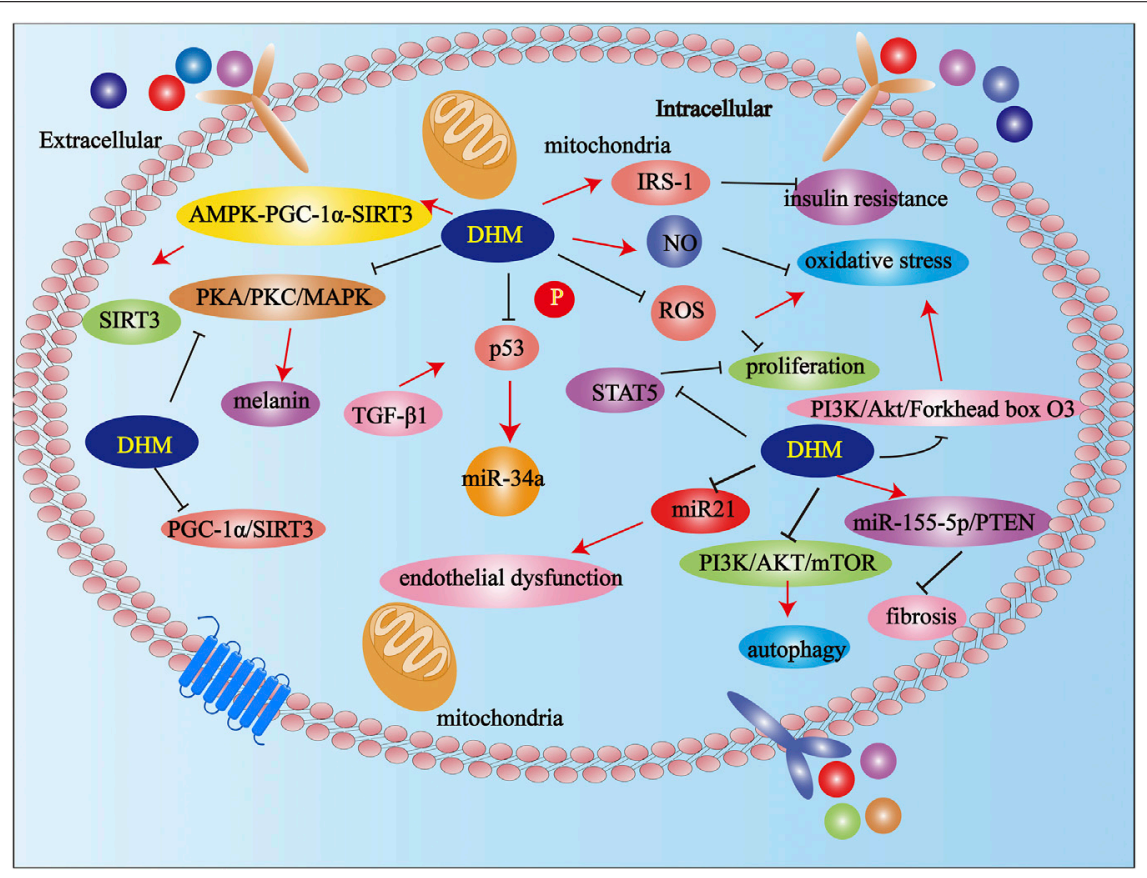

FIGURE 3 | Possible mechanisms of DHM in others expect anti-inflammation. Abbreviations: DHM, Dihydromyricetin; NO, nitric oxide; AKT, protein kinase B; PI3K, Phosphatidylinositol 3-kinase; PGC-1 $\alpha$, peroxisome proliferator-activated receptor $\gamma$ coactiva-tor-1; IRS-1, ginsulin receptor substrate-1; miR-34a, microRNA-34a; ROS, reactive oxygen species. 


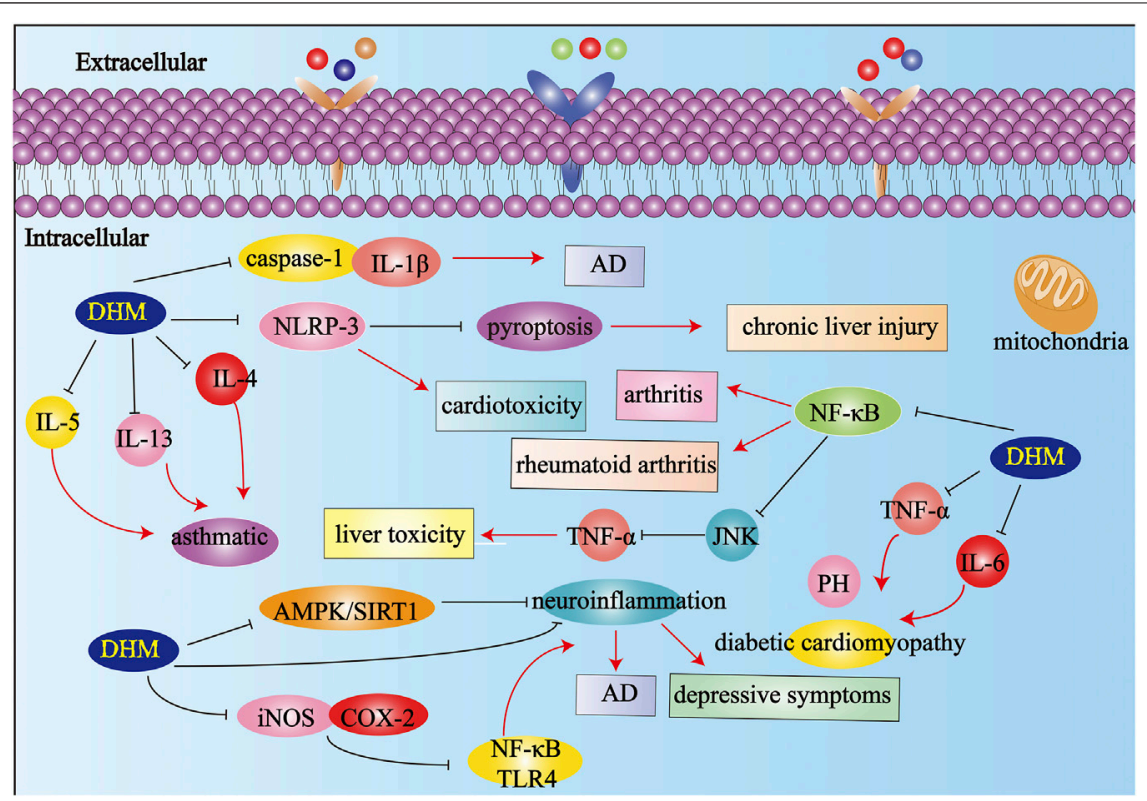

FIGURE 4 | The effects and mechanisms on different inflammatory diseases. Abbreviations: AD, Alzheimer's disease; PH, pulmonary hypertension; IL-1 $\beta$, Interleukin-1 $\beta$; IL-5, Interleukin-5; IL-13, Interleukin-13; IL-4, Interleukin-4; IL-6, Interleukin-6; iNOS, inducible nitric oxide synthase; TNF- $\alpha$, tumor necrosis factor- $\alpha$; COX2, cyclooxygenase-2; JNK, c-Jun N-terminal kinase; AMPK, Adenosine 5'-monophosphate activated protein; NF-kB, Nuclear factor-kB; DHM, Dihydromyricetin; SIRT, Sirtuin.

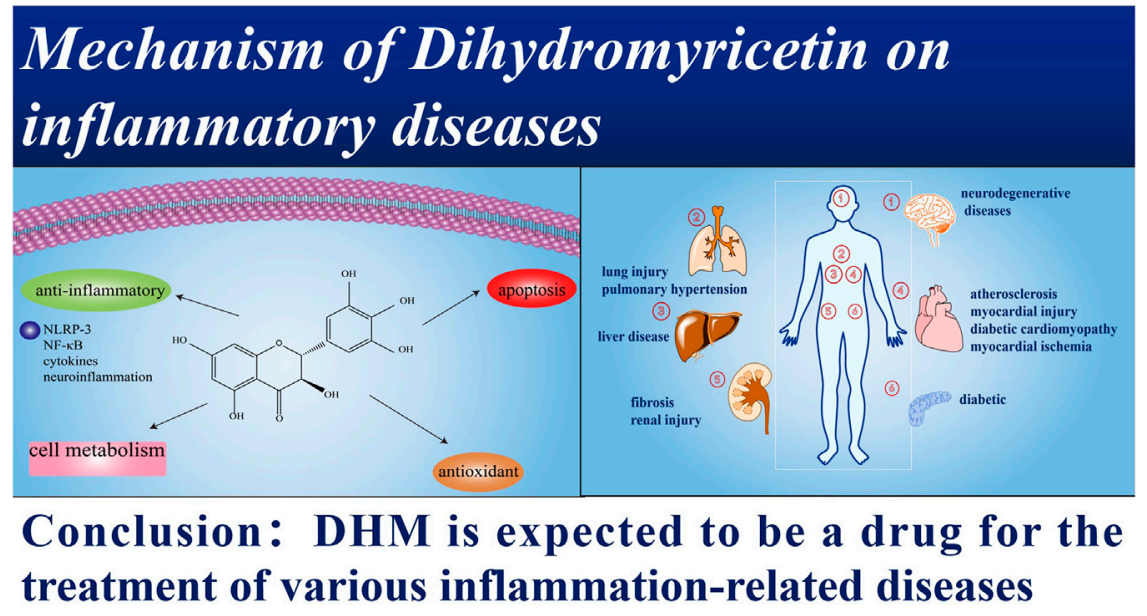

FIGURE 5 | DHM is expected to be a drug for the treatment of various inflammation-related diseases.

et al., 2021). DHM improved emodin-induced hepatotoxicity by inhibiting oxidative stress via the Nrf2 signal pathway in human hepatocyte cell line L02 ${ }^{74}$. Studies have confirmed that DHM improved gentamicin-induced ototoxicity through the PGC-1 $\alpha /$ SIRT3 signaling pathway in house ear institute-organ of corti (HEI-OC)1 (Han et al., 2020). DHM promoted autophagy and improved renal interstitial fibrosis in diabetic nephropathy (DN) by regulating the miR-155-5p/phosphatase and tensin homolog deleted on chromosome ten (PTEN) signaling pathway and phosphatidylinositol 3-kinase (PI3K)/protein kinase B (AKT)/ mammalian target of rapamycin (mTOR) signaling pathway (Guo et al., 2020a). Studies have shown that DHM improved TNF- $\alpha$-induced endothelial dysfunction by inhibiting miR-21 ${ }^{77}$. DHM improved oxidative stress in endothelial cells through PI3K/Akt/Forkhead box O3 (FoxO3a) pathway (Zhang et al., 2019). DHM significantly changed the richness and diversity of the intestinal microbiota and regulated the composition of the intestinal microbiota (Fan et al., 2018). Studies have shown that long-term use of DHM attenuated the development of PD-like behaviors and pathological phenotypes (Guo et al., 2020b). In 
addition, DHM is also a new type of anti-alcoholism drug (Shen et al., 2012). DHM improved liver function and brain histopathology in mice with acute liver failure related the hepatic encephalopathy (Cheng et al., 2021). In addition, DHM also has anti-thrombotic effects (Chen et al., 2021a) (Figure 3).

\section{POSSIBLE CROSSTALK BETWEEN ANTI-INFLAMMATORY AND OTHER EFFECTS IN DHM}

DHM improved TNF- $\alpha$-induced endothelial dysfunction by inhibiting miR-21 (Yang et al., 2018). We speculated whether DHM inhibited a series of inflammatory responses induced by TNF- $\alpha$ by inhibiting the expression of miR-21. DHM inhibited inflammatory response via up-regulation of the AMPK/SIRT1 pathway in AD mice (Sun et al., 2019). DHM inhibited neuroinflammation in $\mathrm{AD}$ rats through $\mathrm{AMPK} / \mathrm{SIRT} 1$ pathway (Liu et al., 2020). This suggested that in addition to the antiinflammatory pathway, DHM mediated other pathways to play an anti-inflammatory role in inflammatory diseases. DHM exerted other anti-inflammatory effects through antiinflammation. On the contrary, DHM exerted antiinflammatory effects via other related signaling pathways. This is an exciting and interesting crosstalk, which is worthy of indepth exploration and discovery in future research. We can knock out an inflammatory gene by gene knockdown and observe the changes of other pathways after DHM treatment. Or more attention should be paid to other related pathways in the study of inflammatory diseases in DHM.

\section{CLINICAL STUDY OF DIHYDROMYRICETIN}

We consulted the studies on DHM in the most recent 20 years, and we found that the clinical reports on DHM were very limited. There were few studies about the anti-inflammatory effect of DHM in the clinic. Sixty adult patients with nonalcoholic fatty liver disease underwent a randomized double-blind experiment. Following DHM treatment, the level of TNF- $\alpha$ and cytokeratin18 in serum decreased significantly in this group (Chen et al., 2021b). In a randomized double-blind trial of 80 patients with type two diabetes for 1 month, data showed that DHM supplementation significantly improved renal function parameters and glycemic control in patients with type two diabetes mellitus (Ran et al., 2019). We should pay more attention to DHM in clinical practice. We should explore the mechanism of DHM through animal and cell experiments to solve the unsolved problems in the clinic.

\section{CHALLENGES AND DIFFICULTIES OF DIHYDROMYRICETIN IN CLINIC}

Studies have shown that DHM is only $0.2 \mathrm{mg} / \mathrm{ml}$ at $25^{\circ} \mathrm{C}$, the solubility of DHM is very low, so DHM cannot be completely absorbed from the intestine (Ran et al., 2019). Xiang et al. evaluated the passive diffusion absorption capacity of DHM through human internal Caco- 2 cells. The results showed that the passive diffusion absorption capacity of DHM was very poor, and the uptake and transport of DMY depended on time and concentration. $\mathrm{PH}$ value affects DMY uptake but not its two-way transport (Xiang et al., 2018). Because of its poor bioavailability and absorption capacity, its clinical application is limited (Ran et al., 2019). Thus far, many studies have reported that DHM inhibited the proliferation of many types of human tumor cells, such as human cholangiocarcinoma cells (Chen et al., 2020), human intestinal Caco-2 cells (Xiang et al., 2018), human ovarian cancer cells (Wang et al., 2019), and human myelomonocytic lymphoma cells (Feng et al., 2019), and so on. However, there are few studies on anti-tumor or else pharmacological effects in vivo or clinic. To better apply DHM in the clinic, improving the bioavailability and gastrointestinal absorption of DHM is not only an urgent problem to be solved but also difficult and challenging for the clinical application of DHM.

\section{DISCUSSION}

Numerous studies have shown that inflammation plays a vital role in a variety of diseases, including diabetes, arthritis, asthma (Zhong and Shi, 2019), AD, acute cerebral stroke, cancer, hypertension, and myocardial ischemia (Liu et al., 2019a; Figure 4). Therefore, the further elucidation of inflammation-related mechanisms and the development of anti-inflammatory drugs are urgent problems to be solved. DHM has been used to treat different diseases for a long time (Martínez-Coria et al., 2019). This review summarizes the mechanism of DHM in inflammatory diseases according to its different effects systematically, focusing on the research progress of DHM in anti-inflammatory, apoptosis, oxidative stress, and the effects of various metabolic pathways. In summary, many research results show that DHM as a component of natural medicine has a variety of pharmacological effects. As we all know, numerous diseases are closely related to inflammation (Medzhitov, 2008). Therefore, we should pay more attention to the research and development of anti-inflammatory mechanisms and anti-inflammatory drugs. DHM has multiple pharmacological effects, especially anti-inflammatory pharmacological effects. However, the specific mechanism and many targets of DHM's anti-inflammatory pharmacological effects need to be further studied and explored. Although a large amount of literature has prompted us, DHM is a potential drug for the treatment of inflammation-related diseases (Table 1). Thus far, most studies on DHM have focused on animal and cell levels, and it is not common for clinical studies. Although studies have confirmed that DHM can inhibit the proliferation of a variety of human tumor cells, it is still limited to the cell level. This is a problem that demands us to think and solve. In future research, we should pay attention to the clinical 
TABLE 1 | The summary of mechanisms of DHM. The latest research progress and related mechanisms of DHM in animals, cells, and clinic were summarized

\begin{tabular}{|c|c|c|}
\hline Experimental model/patients & Mechanism & References \\
\hline AD mice & Down-regulation of caspase- $1, \mathrm{IL}-1 \beta$, and NLRP-3 expression & Feng et al. (2018) \\
\hline Chronic liver injury mice & Down-regulation of NLRP-3 expression & Cheng et al. (2020) \\
\hline SIRT3 ko mice & Down-regulation of NLRP-3 and oxidative stress & Liu et al. (2019b) \\
\hline Myocardial injury rat & Down-regulation of NLRP-3 expression & Sun et al. (2020) \\
\hline Arthritis rats & Down-regulation of NF-kB expression & Wu et al. (2019a) \\
\hline Rheumatoid arthritis rat & Down-regulation of P-NF-kB expression & Wu et al. (2020) \\
\hline LDL receptor-deficient mice & Down-regulation of TNF- $\alpha$ and IL-6 expression & Liu et al. (2017b) \\
\hline Rheumatoid arthritis rat & $\begin{array}{l}\text { Down-regulation of TNF- } \alpha, I L-6, I L-1 \beta \text {, and COX-2 expression } \\
\text { Up-regulation of Nrf2 expression }\end{array}$ & Chu et al. (2018) \\
\hline Liver damage mice & Down-regulation of TNF- $\alpha$ and JNK expression & Xie et al. (2015) \\
\hline Diabetic cardiomyopathy mice & Down-regulation of TNF- $\alpha$ and IL-6 expression & Wu et al. (2017) \\
\hline Pulmonary hypertension rat & Down-regulation of TNF- $\alpha$ and IL-6 expression & Li et al. (2017) \\
\hline Asthmatic mice & Down-regulation of IL-4, IL-5, and IL-13 expression & Xu et al. (2017) \\
\hline $\mathrm{AD}$ rat & Inhibition of AMPK/SIRT1 signal pathway & (Liu et al., 2020), (Sun et al., 2019) \\
\hline Acute liver injury mice & Down-regulation of JNK expression & Wang et al. (2018a) \\
\hline Obese mice & Up-regulation of IRS-1 expression & He et al. (2019) \\
\hline Osteoarthritis rat & Down-regulation of SIRT3 expression & Wang et al. (2018b) \\
\hline HUVECS & $\begin{array}{l}\text { Up-regulation of Nrf2 expression } \\
\text { Down-regulation of NLRP-3 and ROS expression }\end{array}$ & Hu et al. (2018) \\
\hline B16F10 cells & Down-regulation of PKA, PKC, MAPK, and melanin expression & Huang et al. (2016) \\
\hline Tubular Epithelial Cells & Down-regulation of miR-34a expression & Liu et al. (2019c) \\
\hline Endothelial cells & $\begin{array}{l}\text { Down-regulation of ROS expression } \\
\text { Up-regulation of NO expression }\end{array}$ & Hou et al. (2015b) \\
\hline Mast cell & Down-regulation of STAT5 and NF-кB expression & Chang et al. (2021) \\
\hline L02 cell & Up-regulation of Nrf2 expression & Yan et al. (2019) \\
\hline Endothelial cells & Inhibition of PI3K/Akt/FoxO3a signal pathway & Guo et al. (2020a) \\
\hline Patients with renal fibrosis & Down-regulation of miR-34a expression & Liu et al. (2019c) \\
\hline $\begin{array}{l}\text { Patients with nonalcoholic fatty liver } \\
\text { patients with type two diabetes mellitus }\end{array}$ & $\begin{array}{l}\text { Down-regulation of TNF- } \alpha \text { and cytokeratin-18 expression } \\
\text { Down-regulation of glycemic expression }\end{array}$ & $\begin{array}{l}\text { Chen et al. (2021b) } \\
\text { Ran et al. (2019) }\end{array}$ \\
\hline
\end{tabular}

Abbreviations: AD, Alzheimer's disease; COX-2, cyclooxygenase-2; House Ear Institute-Organ of Corti, HEl-OC; HUVECs, human umbilical vein endothelial cells; IL-1 $\beta$, Interleukin-1 $\beta$; IL13, Interleukin-13; IL-4, Interleukin-4; IL-5, Interleukin-5; IL-6, Interleukin-6; IRS-1, ginsulin receptor substrate-1; JNK, c-Jun N-terminal kinase; LDL, Iow-density lipoprotein; L02, human hepatocyte cell; miR-34a, microRNA-34a; NF- $\kappa B$, Nuclear factor- $\kappa B$; NO, nitric oxide; Nrf2, NF-E2-related factor 2; PGC-1 $\alpha$, peroxisome proliferator-activated receptor $\gamma$ coactiva-tor-1; $P-N F-\kappa B$, Phosphorylation-nuclear factor- $\kappa B$; STAT5, signal transducer and activator of transcription 5; ROS, reactive oxygen species; Sirtuin3, SIRT3; TNF- $\alpha$, tumor necrosis factor- $\alpha$

transformation of DHM. The bioavailability and chemical stability of DHM can be improved by changing the dosage form into the sustained-release mechanism, controlled-release preparation, or targeted preparation. DHM is an active compound mainly derived from A.grossedentata. It can also be combined with other related drugs to make compound preparations to improve the curative effect and bioavailability.

The clinical transformation of candidate drugs or active compounds is an urgent problem to be solved in future research, which is meaningful and valuable life science research (Liu et al., 2019d; Figure 5).

\section{AUTHOR CONTRIBUTIONS}

Y.S., S.L., and S.Y. wrote the paper. C.C., Y.Y., M.L., C.L., W.W., and X.Z. modified and submitted the manuscript. Q.A., W.W.,

\section{REFERENCES}

Alessandri, A. L., Sousa, L. P., Lucas, C. D., Rossi, A. G., Pinho, V., and Teixeira, M. M. (2013). Resolution of Inflammation: Mechanisms and Opportunity for Drug Development. Pharmacol. Ther. 139 (2), 189-212. doi:10.1016/ j.pharmthera.2013.04.006 and N.C. guided the manuscript. All authors have read and agreed to the published version of the manuscript.

\section{FUNDING}

This study was supported by Special Scientific and Technological Project for Comprehensive Utilization of Ampelopsis grossedentata Resources of Hunan Qiankun Biotechnology Co., Ltd., (212010), Key Project of Hunan University of Chinese Medicine School Level Scientific Research Fund (2019xjij001), Hunan University of Chinese Medicine FirstClass Disciple Construction Project of Chinese Material Medica and The key discipline of biological engineering of Hunan University of Chinese Medicine (2018) No.3. Pharmaceutical Open Fund of Domestic First-class Disciplines (cultivation) of Hunan Province.

Arulselvan, P., Fard, M. T., Tan, W. S., Gothai, S., Fakurazi, S., Norhaizan, M. E., et al. (2016). Role of Antioxidants and Natural Products in Inflammation. Oxid Med. Cel Longev 2016, 5276130. doi:10.1155/2016/ 5276130

Bai, B., Yang, Y., Wang, Q., Li, M., Tian, C., Liu, Y., et al. (2020). NLRP3 Inflammasome in Endothelial Dysfunction. Cell Death Dis 11, 776. doi:10.1038/s41419-020-02985-x 
Baune, B. T. (2015). Inflammation and Neurodegenerative Disorders: Is There Still hope for Therapeutic Intervention. Curr. Opin. Psychiatry 28 (2), 148-154. doi:10.1097/YCO.0000000000000140

Calsolaro, V., and Edison, P. (2016). Neuroinflammation in Alzheimer's Disease: Current Evidence and Future Directions. Alzheimers Dement 12, 719-732. doi:10.1016/j.jalz.2016.02.010

Cas, M. D., Roda, G., Li, F., and Secundo, F. (2020). Functional Lipids in Autoimmune Inflammatory Diseases. Int. J. Mol. Sci. 21 (9), 3074. doi:10.3390/ijms21093074

Chang, T. M., Hsiao, T. C., Yang, T. Y., and Huang, H. C. (2021). IgE-Induced Mast Cell Activation Is Suppressed by Dihydromyricetin through the Inhibition of NF-Kb Signaling Pathway. Molecules 26, 3877. doi:10.3390/molecules26133877

Chen, J., Wu, Y., Zou, J., and Gao, K. (2016). $\alpha$-Glucosidase Inhibition and Antihyperglycemic Activity of Flavonoids from Ampelopsis Grossedentata and the Flavonoid Derivatives. Bioorg. Med. Chem. 24, 1488-1494. doi:10.1016/j.bmc.2016.02.018

Chen, L., Yang, Z. S., Zhou, Y. Z., Deng, Y., Jiang, P., and Tan, S. L. (2020). Dihydromyricetin Inhibits Cell Proliferation, Migration, Invasion and Promotes Apoptosis via Regulating miR-21 in Human Cholangiocarcinoma Cells. J. Cancer 11 (19), 5689-5699. doi:10.7150/jca.45970

Chen, S., Lv, K., Sharda, A., Deng, J., Zeng, W., Zhang, C., et al. (2021). Antithrombotic Effects Mediated by Dihydromyricetin Involve Both Platelet Inhibition and Endothelial protection. Pharmacol. Res. 167, 105540. doi:10.1016/j.phrs.2021.105540

Chen, S., Lv, K., Sharda, A., Deng, J., Zeng, W., Zhang, C., et al. (2021). Antithrombotic Effects Mediated by Dihydromyricetin Involve Both Platelet Inhibition and Endothelial protection. Pharmacol. Res. 167, 105540. doi:10.1016/j.phrs.2021.105540

Cheng, L., Wang, X., Ma, X., Xu, H., Yang, Y., and Zhang, D. (2021). Effect of Dihydromyricetin on Hepatic Encephalopathy Associated with Acute Hepatic Failure in Mice. Pharm. Biol. 59, 557-564. doi:10.1080/13880209.2021.1917625

Cheng, Q. C., Fan, J., Deng, X. W., Liu, H. C., Ding, H. R., Fang, X., et al. (2020). Dihydromyricetin Ameliorates Chronic Liver Injury by Reducing Pyroptosis. World J. Gastroenterol. 26 (41), 6346-6360. doi:10.3748/wjg.v26.i41.6346

Chu, J., Wang, X., Bi, H., Li, L., Ren, M., and Wang, J. (2018). Dihydromyricetin Relieves Rheumatoid Arthritis Symptoms and Suppresses Expression of Proinflammatory Cytokines via the Activation of Nrf2 Pathway in Rheumatoid Arthritis Model. Int. Immunopharmacol 59, 174-180. doi:10.1016/ j.intimp.2018.04.001

Dhingra, A. K., and Chopra, B. (2020). Inflammation as a Therapeutic Target for Various Deadly Disorders: A Review. Curr. Drug Targets 21 (6), 582-588. doi:10.2174/1389450120666191204154115

Diakos, C. I., Charles, K. A., McMillan, D. C., and Clarke, S. J. (2014). Cancerrelated Inflammation and Treatment Effectiveness. Lancet Oncol. 15, e493-503. doi:10.1016/S1470-2045(14)70263-3

Ding, Y., Gong, W., Zhang, S., Shen, J., Liu, X., Wang, Y., et al. (2021). Protective Role of Sirtuin3 against Oxidative Stress and NLRP3 Inflammasome in Cholesterol Accumulation and Foam Cell Formation of Macrophages with Ox-LDL-Stimulation. Biochem. Pharmacol. 192, 114665. doi:10.1016/ j.bcp.2021.114665

Fan, L., Zhao, X., Tong, Q., Zhou, X., Chen, J., Xiong, W., et al. (2018). Interactions of Dihydromyricetin, a Flavonoid from Vine Tea (Ampelopsis Grossedentata) with Gut Microbiota. J. Food Sci. 83, 1444-1453. doi:10.1111/1750-3841.14128

Feng, J., Wang, J. X., Du, Y. H., Liu, Y., Zhang, W., Chen, J. F., et al. (2018). Dihydromyricetin Inhibits Microglial Activation and Neuroinflammation by Suppressing NLRP3 Inflammasome Activation in APP/PS1 Transgenic Mice. CNS Neurosci. Ther. 24, 1207-1218. doi:10.1111/cns.12983

Feng, Q. W., Cui, Z. G., Jin, Y. J., Sun, L., Li, M. L., Zakki, S. A., et al. (2019). Protective Effect of Dihydromyricetin on Hyperthermia-Induced Apoptosis in Human Myelomonocytic Lymphoma Cells. Apoptosis 24 (3-4), 290-300. doi:10.1007/s10495-019-01518-y

Gao, Q., Ma, R., Chen, L., Shi, S., Cai, P., Zhang, S., et al. (2017). Antioxidant Profiling of Vine tea (Ampelopsis Grossedentata): Off-Line Coupling HeartCutting HSCCC with HPLC-DAD-QTOF-MS/MS. Food Chem. 225, 55-61. doi:10.1016/j.foodchem.2016.11.122

Geovanini, G. R., and Libby, P. (2018). Atherosclerosis and Inflammation: Overview and Updates. Clin. Sci. (Lond) 132, 1243-1252. doi:10.1042/ CS20180306
Guo, C. H., Cao, T., Zheng, L. T., Waddington, J. L., and Zhen, X. C. (2020). Development and Characterization of an Inducible Dicer Conditional Knockout Mouse Model of Parkinson's Disease: Validation of the Antiparkinsonian Effects of a Sigma-1 Receptor Agonist and Dihydromyricetin. Acta Pharmacol. Sin 41, 499-507. doi:10.1038/s41401020-0379-5

Guo, L., Tan, K., Luo, Q., and Bai, X. (2020). Dihydromyricetin Promotes Autophagy and Attenuates Renal Interstitial Fibrosis by Regulating miR155-5p/PTEN Signaling in Diabetic Nephropathy. Bosn J. Basic Med. Sci. 20, 372-380. doi:10.17305/bjbms.2019.4410

Han, H., Dong, Y., and Ma, X. (2020). Dihydromyricetin Protects against Gentamicin-Induced Ototoxicity via PGC-1 $\alpha /$ SIRT3 Signaling In Vitro. Front Cel Dev Biol 8, 702. doi:10.3389/fcell.2020.00702

He, F., Antonucci, L., and Karin, M. (2020). NRF2 as a Regulator of Cell Metabolism and Inflammation in Cancer. Carcinogenesis 41 (4), 405-416. doi:10.1093/carcin/bgaa039

He, J., Zhang, J., Dong, L., Dang, X., Wang, L., Cheng, L., et al. (2019). Dihydromyricetin Attenuates Metabolic Syndrome and Improves Insulin Sensitivity by Upregulating Insulin Receptor Substrate-1 (Y612) Tyrosine Phosphorylation in $\mathrm{Db} / \mathrm{db}$ Mice. Diabetes Metab. Syndr. Obes. 12, 2237-2249. doi:10.2147/DMSO.S218487

Hou, X. L., Tong, Q., Wang, W. Q., Shi, C. Y., Xiong, W., Chen, J., et al. (2015). Suppression of Inflammatory Responses by Dihydromyricetin, a Flavonoid from Ampelopsis Grossedentata, via Inhibiting the Activation of NF-Kb and MAPK Signaling Pathways. J. Nat. Prod. 78, 1689-1696. doi:10.1021/ acs.jnatprod.5b00275

Hou, X., Tong, Q., Wang, W., Xiong, W., Shi, C., and Fang, J. (2015). Dihydromyricetin Protects Endothelial Cells from Hydrogen PeroxideInduced Oxidative Stress Damage by Regulating Mitochondrial Pathways. Life Sci. 130, 38-46. doi:10.1016/j.lfs.2015.03.007

Hu, H., Luo, F., Wang, M., Fu, Z., and Shu, X. (2020). New Method for Extracting and Purifying Dihydromyricetin from Ampelopsis Grossedentata. ACS Omega 5, 13955-13962. doi:10.1021/acsomega.0c01222

Hu, Q., Zhang, T., Yi, L., Zhou, X., and Mi, M. (2018). Dihydromyricetin Inhibits NLRP3 Inflammasome-dependent Pyroptosis by Activating the Nrf2 Signaling Pathway in Vascular Endothelial Cells. Biofactors 44 (2), 123-136. doi:10.1002/ biof.1395

Huang, B., Li, Y., Yao, Y., Shu, W., and Chen, M. (2018). Dihydromyricetin from Ampelopsis Grossedentata Protects against Vascular Neointimal Formation via Induction of TR3. Eur. J. Pharmacol. 838, 23-31. doi:10.1016/ j.ejphar.2018.09.002

Huang, H. C., Liao, C. C., Peng, C. C., Lim, J. M., Siao, J. H., Wei, C. M., et al. (2016). Dihydromyricetin from Ampelopsis Grossedentata Inhibits Melanogenesis through Down-Regulation of MAPK, PKA and PKC Signaling Pathways. Chem. Biol. Interact 258, 166-174. doi:10.1016/j.cbi.2016.08.023

Jayaraj, R. L., Azimullah, S., Beiram, R., Jalal, F. Y., and Rosenberg, G. A. (2019). Neuroinflammation: Friend and Foe for Ischemic Stroke. J. Neuroinflammation 16, 142. doi:10.1186/s12974-019-1516-2

Jia, C., Zhang, M., Ma, W., Li, J., Zhao, S., Xiong, S., et al. (2020). Evaluation of Antioxidant Properties of the Different Tissues of Vine tea ( Ampelopsis Grossedentata ) in Stripped Canola Oil and sunflower Oil. J. Food Sci. 85, 1082-1089. doi:10.1111/1750-3841.15092

Jing, N., and Li, X. (2019). Dihydromyricetin Attenuates Inflammation through TLR4/NF-kappaB Pathway. Open Med. (Wars) 14, 719-725. doi:10.1515/med2019-0083

Kirkpatrick, B., and Miller, B. J. (2013). Inflammation and Schizophrenia. Schizophr Bull. 39 (6), 1174-1179. doi:10.1093/schbul/sbt141

Kustrimovic, N., Marino, F., and Cosentino, M. (2019). Peripheral Immunity, Immunoaging and Neuroinflammation in Parkinson's Disease. Curr. Med. Chem. 26 (20), 3719-3753. doi:10.2174/0929867325666181009161048

Li, L., Luo, W., Qian, Y., Zhu, W., Qian, J., Li, J., et al. (2019). Luteolin Protects against Diabetic Cardiomyopathy by Inhibiting NF-Kb-Mediated Inflammation and Activating the Nrf2-Mediated Antioxidant Responses. Phytomedicine 59, 152774. doi:10.1016/j.phymed.2018.11.034

Li, Q., Wang, J., Zhu, X., Zeng, Z., Wu, X., Xu, Y., et al. (2017). Dihydromyricetin Prevents Monocrotaline-Induced Pulmonary Arterial Hypertension in Rats. Biomed. Pharmacother. 96, 825-833. doi:10.1016/ j.biopha.2017.10.007 
Li, X., Cao, M., Ma, W., Jia, C., Li, J., Zhang, M., et al. (2020). Annotation of Genes Involved in High Level of Dihydromyricetin Production in Vine tea (Ampelopsis Grossedentata) by Transcriptome Analysis. BMC Plant Biol. 20, 131. doi:10.1186/s12870-020-2324-7

Liang, H., He, K., Li, T., Cui, S., Tang, M., Kang, S., et al. (2020). Mechanism and Antibacterial Activity of Vine tea Extract and Dihydromyricetin against Staphylococcus aureus. Sci. Rep. 10 (1), 21416. doi:10.1038/s41598-02078379-y

Liang, J., Shen, Y., Shao, X. M., Scott, M. B., Ly, E., Wong, S., et al. (2014). Dihydromyricetin Prevents Fetal Alcohol Exposure-Induced Behavioral and Physiological Deficits: the Roles of GABAA Receptors in Adolescence. Neurochem. Res. 39, 1147-1161. doi:10.1007/s11064-014-1291-5

Liang, X., Wu, Y. P., Qiu, J. H., Zhong, K., and Gao, H. (2014). A Potent Antibrowning Agent from pine needles of Cedrus Deodara: 2R,3RDihydromyricetin. J. Food Sci. 79, C1643-C1648. doi:10.1111/1750-3841.12583

Liu, D., Mao, Y., Ding, L., and Zeng, X. A. (2019). Dihydromyricetin: A Review on Identification and Quantification Methods, Biological Activities, Chemical Stability, Metabolism and Approaches to Enhance its Bioavailability. Trends Food Sci. Technol. 91, 586-597. doi:10.1016/j.tifs.2019.07.038

Liu, D., Mao, Y., Ding, L., and Zeng, X. A. (2019). Dihydromyricetin: A Review on Identification and Quantification Methods, Biological Activities, Chemical Stability, Metabolism and Approaches to Enhance its Bioavailability. Trends Food Sci. Technol. 91, 586-597. doi:10.1016/j.tifs.2019.07.038

Liu, D., Mao, Y., Ding, L., and Zeng, X. A. (2019). Dihydromyricetin: A Review on Identification and Quantification Methods, Biological Activities, Chemical Stability, Metabolism and Approaches to Enhance its Bioavailability. Trends Food Sci. Technol. 91, 586-597. doi:10.1016/j.tifs.2019.07.038

Liu, J., and Wang, F. (2017). Role of Neuroinflammation in Amyotrophic Lateral Sclerosis: Cellular Mechanisms and Therapeutic Implications. Front. Immunol. 8, 1005. doi:10.3389/fimmu.2017.01005

Liu, L., Yin, X., Wang, X., and Li, X. (2017). Determination of Dihydromyricetin in Rat Plasma by LC-MS/MS and its Application to a Pharmacokinetic Study. Pharm. Biol. 55 (1), 657-662. doi:10.1080/13880209.2016.1266669

Liu, M., Guo, H., Li, Z., Zhang, C., Zhang, X., Cui, Q., et al. (2020). Molecular Level Insight into the Benefit of Myricetin and Dihydromyricetin Uptake in Patients with Alzheimer's Diseases. Front. Aging Neurosci. 12, 601603. doi:10.3389/ fnagi.2020.601603

Liu, P., Zou, D., Chen, K., Zhou, Q., Gao, Y., Huang, Y., et al. (2016). Dihydromyricetin Improves Hypobaric Hypoxia-Induced Memory Impairment via Modulation of SIRT3 Signaling. Mol. Neurobiol. 53, 7200-7212. doi:10.1007/s12035-015-9627-y

Liu, T. T., Zeng, Y., Tang, K., Chen, X., Zhang, W., and Xu, X. L. (2017). Dihydromyricetin Ameliorates Atherosclerosis in LDL Receptor Deficient Mice. Atherosclerosis 262, 39-50. doi:10.1016/j.atherosclerosis.2017.05.003

Liu, Y., Bi, X., Xiong, J., Han, W., Xiao, T., Xu, X., et al. (2019). MicroRNA-34a Promotes Renal Fibrosis by Downregulation of Klotho in Tubular Epithelial Cells. Mol. Ther. 27, 1051-1065. doi:10.1016/j.ymthe.2019.02.009

Lopez-Castejon, G. (2020). Control of the Inflammasome by the Ubiquitin System. FEBS J. 287 (1), 11-26. doi:10.1111/febs.15118

Ma, Q., Cai, S., Jia, Y., Sun, X., Yi, J., and Du, J. (2020). Effects of Hot-Water Extract from Vine Tea (Ampelopsis Grossedentata) on Acrylamide Formation, Quality and Consumer Acceptability of Bread. Foods 9, 373. doi:10.3390/foods 9030373

Martínez-Coria, H., Mendoza-Rojas, M. X., Arrieta-Cruz, I., and López-Valdés, H. E. (2019). Preclinical Research of Dihydromyricetin for Brain Aging and Neurodegenerative Diseases. Front. Pharmacol. 10, 1334. doi:10.3389/ fphar.2019.01334

Medzhitov, R. (2008). Origin and Physiological Roles of Inflammation. Nature 454, 428-435. doi:10.1038/nature07201

Ran, L., Wang, X., Lang, H., Xu, J., Wang, J., Liu, H., et al. (2019). Ampelopsis Grossedentata Supplementation Effectively Ameliorates the Glycemic Control in Patients with Type 2 Diabetes Mellitus. Eur. J. Clin. Nutr. 73 (5), 776-782. doi:10.1038/s41430-018-0282-z

Ren, Z., Yan, P., Zhu, L., Yang, H., Zhao, Y., Kirby, B. P., et al. (2018). Dihydromyricetin Exerts a Rapid Antidepressant-like Effect in Association with Enhancement of BDNF Expression and Inhibition of Neuroinflammation. Psychopharmacology (Berl) 235, 233-244. doi:10.1007/s00213-017-4761-z

Sgambato, A., and Cittadini, A. (2010). Inflammation and Cancer: a Multifaceted Link. Eur. Rev. Med. Pharmacol. Sci. 14 (4), 263-268.
Shen, Y., Lindemeyer, A. K., Gonzalez, C., Shao, X. M., Spigelman, I., Olsen, R. W., et al. (2012). Dihydromyricetin as a Novel Anti-alcohol Intoxication Medication. J. Neurosci. 32, 390-401. doi:10.1523/JNEUROSCI.4639-11.2012

Shirazi, L. F., Bissett, J., Romeo, F., and Mehta, J. L. (2017). Role of Inflammation in Heart Failure. Curr. Atheroscler. Rep. 19 (6), 27. doi:10.1007/s11883-0170660-3

Stephenson, J., Nutma, E., van der Valk, P., and Amor, S. (2018). Inflammation in CNS Neurodegenerative Diseases. Immunology 154 (2), 204-219. doi:10.1111/ imm. 12922

Sun, C. C., Li, Y., Yin, Z. P., and Zhang, Q. F. (2021). Physicochemical Properties of Dihydromyricetin and the Effects of Ascorbic Acid on its Stability and Bioavailability. J. Sci. Food Agric. 101 (9), 3862-3869. doi:10.1002/jsfa.11022

Sun, P., Yin, J. B., Liu, L. H., Guo, J., Wang, S. H., Qu, C. H., et al. (2019). Protective Role of Dihydromyricetin in Alzheimer's Disease Rat Model Associated with Activating AMPK/SIRT1 Signaling Pathway. Biosci. Rep. 39, BSR20180902. doi:10.1042/BSR20180902

Sun, Z., Lu, W., Lin, N., Lin, H., Zhang, J., Ni, T., et al. (2020). Dihydromyricetin Alleviates Doxorubicin-Induced Cardiotoxicity by Inhibiting NLRP3 Inflammasome through Activation of SIRT1. Biochem. Pharmacol. 175, 113888. doi:10.1016/j.bcp.2020.113888

Tang, N., Ma, J., Wang, K. S., Mi, C., Lv, Y., Piao, L. X., et al. (2016). Dihydromyricetin Suppresses TNF- $\alpha$-Induced NF-Kb Activation and Target Gene Expression. Mol. Cel Biochem 422, 11-20. doi:10.1007/s11010-0162799-6

Tong, Q., Hou, X., Fang, J., Wang, W., Xiong, W., Liu, X., et al. (2015). Determination of Dihydromyricetin in Rat Plasma by LC-MS/MS and its Application to a Pharmacokinetic Study. J. Pharm. Biomed. Anal. 114, 455-461. doi:10.1016/j.jpba.2015.06.030

Troubat, R., Barone, P., Leman, S., Desmidt, T., Cressant, A., Atanasova, B., et al. (2021). Neuroinflammation and Depression: A Review. Eur. J. Neurosci. 53, 151-171. doi:10.1111/ejn.14720

Wang, B., Xiao, Y., Yang, X., He, Y., Jing, T., Wang, W., et al. (2018). Protective Effect of Dihydromyricetin on LPS-Induced Acute Lung Injury. J. Leukoc. Biol. 103, 1241-1249. doi:10.1002/jlb.3ma0317-101rrr

Wang, F., Chen, X., Yuan, D., Yi, Y., and Luo, Y. (2019). Golgi Reassembly and Stacking Protein 65 Downregulation Is Required for the Anti-cancer Effect of Dihydromyricetin on Human Ovarian Cancer Cells. PLoS One 14 (11), e0225450. doi:10.1371/journal.pone.0225450

Wang, J., Wang, K., Huang, C., Lin, D., Zhou, Y., Wu, Y., et al. (2018). SIRT3 Activation by Dihydromyricetin Suppresses Chondrocytes Degeneration via Maintaining Mitochondrial Homeostasis. Int. J. Biol. Sci. 14 (13), 1873-1882. doi:10.7150/ijbs.27746

Wang, R., Pi, J., Su, X., Liu, J., Zeng, X., Wong, I., et al. (2016). Dihydromyricetin Suppresses Inflammatory Responses In Vitro and In Vivo through Inhibition of IKK $\beta$ Activity in Macrophages. Scanning 38, 901-912. doi:10.1002/sca.21339

Wang, R., Pi, J., Su, X., Liu, J., Zeng, X., Wong, I., et al. (2016). Dihydromyricetin Suppresses Inflammatory Responses In Vitro and In Vivo through Inhibition of IKK $\beta$ Activity in Macrophages. Scanning 38, 901-912. doi:10.1002/sca.21339

Wu, B., Lin, J., Luo, J., Han, D., Fan, M., Guo, T., et al. (2017). Dihydromyricetin Protects against Diabetic Cardiomyopathy in Streptozotocin-Induced Diabetic Mice. Biomed. Res. Int. 2017, 3764370. doi:10.1155/2017/3764370

Wu, F., Li, Y., Song, H., Zhang, Y., Zhang, Y., Jiang, M., et al. (2016). Preventive Effect of Dihydromyricetin against Cisplatin-Induced Nephrotoxicity In Vitro and In Vivo. Evid. Based Complement. Alternat Med. 2016, 7937385. doi:10.1155/2016/7937385

Wu, J., Fan, K. J., Wang, Q. S., Xu, B. X., Cai, Q., and Wang, T. Y. (2020). DMY Protects the Knee Joints of Rats with Collagen-Induced Arthritis by Inhibition of NF-Kb Signaling and Osteoclastic Bone Resorption. Food Funct. 11, 6251-6264. doi:10.1039/d0fo00396d

Wu, J. Z., Ardah, M., Haikal, C., Svanbergsson, A., Diepenbroek, M., Vaikath, N. N., et al. (2019). Dihydromyricetin and Salvianolic Acid B Inhibit AlphaSynuclein Aggregation and Enhance Chaperone-Mediated Autophagy. Transl Neurodegener 8, 18. doi:10.1186/s40035-019-0159-7

Wu, J., Zhao, F. T., Fan, K. J., Zhang, J., Xu, B. X., Wang, Q. S., et al. (2019). Dihydromyricetin Inhibits Inflammation of Fibroblast-like Synoviocytes through Regulation of Nuclear Factor-Kb Signaling in Rats with CollagenInduced Arthritis. J. Pharmacol. Exp. Ther. 368, 218-228. doi:10.1124/ jpet.118.253369 
Wu, P., Ma, G., Li, N., Deng, Q., Yin, Y., and Huang, R. (2015). Investigation of In Vitro and In Vivo Antioxidant Activities of Flavonoids Rich Extract from the Berries of Rhodomyrtus tomentosa(Ait.) Hassk. Food Chem. 173, 194-202. doi:10.1016/j.foodchem.2014.10.023

Xiang, D., Fan, L., Hou, X. L., Xiong, W., Shi, C. Y., Wang, W. Q., et al. (2018). Uptake and Transport Mechanism of Dihydromyricetin across Human Intestinal Caco-2 Cells. J. Food Sci. 83 (7), 1941-1947. doi:10.1111/1750-3841.14112

Xie, J., Liu, J., Chen, T. M., Lan, Q., Zhang, Q. Y., Liu, B., et al. (2015). Dihydromyricetin Alleviates Carbon Tetrachloride-Induced Acute Liver Injury via JNK-dependent Mechanism in Mice. World J. Gastroenterol. 21, 5473-5481. doi:10.3748/wjg.v21.i18.5473

Xu, B., Huang, S., Wang, C., Zhang, H., Fang, S., and Zhang, Y. (2017). Antiinflammatory E-ffects of D-ihydromyricetin in a M-ouse M-odel of A-sthma. Mol. Med. Rep. 15, 3674-3680. doi:10.3892/mmr.2017.6428

Yalta, T., and Yalta, K. (2018). Systemic Inflammation and Arrhythmogenesis: A Review of Mechanistic and Clinical Perspectives. Angiology 69 (4), 288-296. doi:10.1177/0003319717709380

Yan, Y., Wang, K., Tang, X., Gao, J. F., and Wen, B. Y. (2019). Phytochemicals Protect L02 Cells against Hepatotoxicity Induced by Emodin via the Nrf2 Signaling Pathway. Toxicol. Res. (Camb) 8, 1028-1034. doi:10.1039/c9tx00220k

Yang, D., Tan, S., Yang, Z., Jiang, P., Qin, C., Yuan, Q., et al. (2018). Dihydromyricetin Attenuates TNF- $\alpha$-Induced Endothelial Dysfunction through miR-21-Mediated DDAH1/ADMA/NO Signal Pathway. Biomed. Res. Int. 2018, 1047810. doi:10.1155/2018/1047810

Yang, Y. M., Kim, S. Y., and Seki, E. (2019). Inflammation and Liver Cancer: Molecular Mechanisms and Therapeutic Targets. Semin. Liver Dis. 39, 26-42. doi:10.1055/s-0038-1676806

Zhang, J., Chen, Y., Luo, H., Sun, L., Xu, M., Yu, J., et al. (2018). Recent Update on the Pharmacological Effects and Mechanisms of Dihydromyricetin. Front. Pharmacol. 9, 1204. doi:10.3389/fphar.2018.01204

Zhang, Q., Liu, J., Liu, B., Xia, J., Chen, N., Chen, X., et al. (2014). Dihydromyricetin Promotes Hepatocellular Carcinoma Regression via a P53 Activationdependent Mechanism. Sci. Rep. 4, 4628. doi:10.1038/srep04628
Zhang, X., Wang, L., Peng, L., Tian, X., Qiu, X., Cao, H., et al. (2019). Dihydromyricetin Protects HUVECs of Oxidative Damage Induced by Sodium Nitroprusside through Activating PI3K/Akt/FoxO3a Signalling Pathway. J. Cel Mol Med 23 (7), 4829-4838. doi:10.1111/jcmm.14406

Zhang, Y., Yang, W., and Xiong, H. (2001). Basic Constituent of Ampelopsis Grossedentata. Nat. Product. Res. Dev. 13, 46-48.

Zhong, J., and Shi, G. (2019). Editorial: Regulation of Inflammation in Chronic Disease. Front. Immunol. 10, 737. doi:10.3389/fimmu.2019.00737

Zhou, Y., Liang, X., Chang, H., Shu, F., Wu, Y., Zhang, T., et al. (2014). Ampelopsin-induced Autophagy Protects Breast Cancer Cells from Apoptosis through Akt-mTOR Pathway via Endoplasmic Reticulum Stress. Cancer Sci. 105, 1279-1287. doi:10.1111/cas.12494

Conflict of Interest: C.L. and W.W. were employed by the company Zhangiiajie Meicha Technology Research Center, Hunan Qiankun Biotechnology Co., Ltd.

The remaining authors declare that the research was conducted in the absence of any commercial or financial relationships that could be construed as a potential conflict of interest.

Publisher's Note: All claims expressed in this article are solely those of the authors and do not necessarily represent those of their affiliated organizations, or those of the publisher, the editors, and the reviewers. Any product that may be evaluated in this article, or claim that may be made by its manufacturer, is not guaranteed or endorsed by the publisher.

Copyright (C) 2022 Sun, Liu, Yang, Chen, Yang, Lin, Liu, Wang, Zhou, Ai, Wang and Chen. This is an open-access article distributed under the terms of the Creative Commons Attribution License (CC BY). The use, distribution or reproduction in other forums is permitted, provided the original author(s) and the copyright owner(s) are credited and that the original publication in this journal is cited, in accordance with accepted academic practice. No use, distribution or reproduction is permitted which does not comply with these terms. 